\title{
A multisensor approach for the 2016 Amatrice earthquake damage as- sessment
}

\author{
VITO ROMANIELLO ${ }^{* 1}$, ALESSANDRO PISCINI ${ }^{1}$, CHRISTIAN \\ BIGNAMI ${ }^{1}$, ROBERTA ANNIBALLE ${ }^{2}$, AND SALVATORE \\ STRAMONDO 1 \\ ${ }^{1}$ Istituto Nazionale di Geofisica e Vulcanologia \\ 2 DIET, Sapienza University of Rome \\ *vito.romaniello@ingv.it
}

\begin{abstract}
This work proposes methodologies aimed at evaluating the damage occurred in the Amatrice town by using optical and Synthetic Aperture Radar (SAR) change features obtained from satellite images. The objective is to achieve a damage map employing the satellite change features in a classifier algorithm, namely the Features Stepwise Thresholding (FST) method. The main novelties of the proposed analysis concern the estimation of derived features at object scale and the exploitation of the unsupervised FST algorithm. A segmentation of the study area into several buildings blocks has been done by considering a set of polygons, over the Amatrice town, extracted from the open source Open Street Map (OSM) geo-database.

The available satellite dataset is composed of several optical and SAR images, collected before and after the seismic event.

Regarding the optical data, we selected the Normalised Difference Index (NDI), and two quantities coming from the Information Theory, namely the Kullback-Libler Divergence (KLD) and the Mutual Information (MI). In addition, for the SAR data we picked out the Intensity Correlation Difference (ICD) and the KLD parameter.

The exploitation of these features in the FST algorithm permits to obtain a plausible damage map that is able to indicate the most affected areas.
\end{abstract}

\section{INTRODUCTION}

$\Lambda$ n earthquake damage map, available right after a seismic event (from few hours up to few days, depending on the satellite data availability), can guide the rescue teams interventions towards the most affected areas. Satellite data can be very useful for this purpose, thanks to the wide coverage and the high spatial resolution, especially to map damage in large regions, where villages cannot easily accessed. Satellite damage assessment is based on detection techniques that are capable to identify changes of an object by observing it at different times (Ingram (1981); Jenson (1983)). Both optical and radar sensors can be exploited for change detection purposes and several examples are reported in the literature 
(Matsuoka and Yamazaki (2004); Hoffman et al. (2004); Yonezawa and Takeuchi (2001); Stramondo et al. (2006); Chini et al. (2013)).

By exploiting both optical and radar sensors, and using change features achieved from the statistical analysis, a more accurate and reliable damage mapping can be obtained. Bignami et al. (2004) have analysed the possible advantages of combining radar and optical satellite data.

Regarding the SAR data, the Intensity Correlation Difference (ICD) demonstrated to be a very good damage proxy (Stramondo et al. (2006)). KLD and MI (Brunner et al. (2010)) are also suitable features that can contribute to damage estimation.

As far as the optical data is concerned, the most significative results are related to the Normalised Difference Index (NDI), KullbackLibler Divergence (KLD) (Kullback and Leiber (1951)), and Mutual Information (MI) indexes (Xie et al. (2003)). All these features show a good sensitivity to the collapse ratio.

In this work, we used the NDI, KLD and MI indexes for the optical data, and the ICD and the KLD parameters for the SAR data, following the outcomes carried out during EU funded project APhoRISM (Advanced PRocedures for volcanic and Seismic Monitoring, www.aphorism-project.eu).

The main novelties of the proposed analysis concern the estimation of derived features at object scale and the use of an unsupervised algorithm for damage estimation purposes. In order to exploit the object-oriented approach, a segmentation of the study area into several regions was performed. In particular, testing an operational approach, we have segmented and then generated the damage map of Amatrice by considering a set of polygons extracted from the open source Open Street Map (OSM) geo-database. Finally, an unsupervised algorithm to estimate the damage level by satellite features, namely in the paper Features Stepwise Thresholding (FST), has been used (Roaniello et al. (2016)).

\section{DATA AND METHODS}

The available dataset is made up of two Sentinel-2 optical images (1 pre- and 1 postseismic), three COSMO-Sky SAR images (2 pre- and 1 post-seismic) and a buildings footprint layer extracted by the Open Street Map service (see Table 1 for EO data characteristics). Both optical and SAR data have a spatial resolution of $10 \mathrm{~m}$.

\begin{tabular}{lll}
\hline Datatype & Satellite & Acquisition \\
\hline Optical & Sentinel-2 & $2016 / 08 / 14$ \\
Optical & Sentinel-2 & $2016 / 09 / 04$ \\
SAR & COSMO-Sky & $2016 / 07 / 19$ \\
SAR & COSMO-Sky & $2016 / 08 / 20$ \\
SAR & COSMO-Sky & $2016 / 08 / 28$ \\
\hline
\end{tabular}

Table 1: EO data list

The Amatrice footprint layer extracted from OSM, which is at single building scale, was modified to obtain polygons surrounding more than one building. In this way, there are more pixels associated to each polygon leading a better estimation of change features over the polygon itself. The resulting layer consist of 112 polygons, inside an area of about $1 \mathrm{~km}^{2}$.

Previous studies, carried out during the European APhoRISM project, allowed us to identify the features having a good sensitivity to damage at object scale (Romaniello et al. (2016)). Following the project outcomes, in the present work we used the NDI, and two quantities coming from the Information Theory, the KLD and the MI. In addition, for the SAR data, we picked out the ICD parameter. 
The NDI parameter is defined as:

$$
N D I_{i}=\frac{P O S T_{i}-P R E_{i}}{P O S T_{i}+P R E_{i}}
$$

Where PREi and POSTi indicate the mean values of intensity, respectively for pre- and post- seismic images, associated to i-th polygon. The NDI parameter was computed, considering pre- and post-seismic Sentinel-2 images, for each polygon obtained from OSM service. Fig. 1 shows the resulting NDI map.
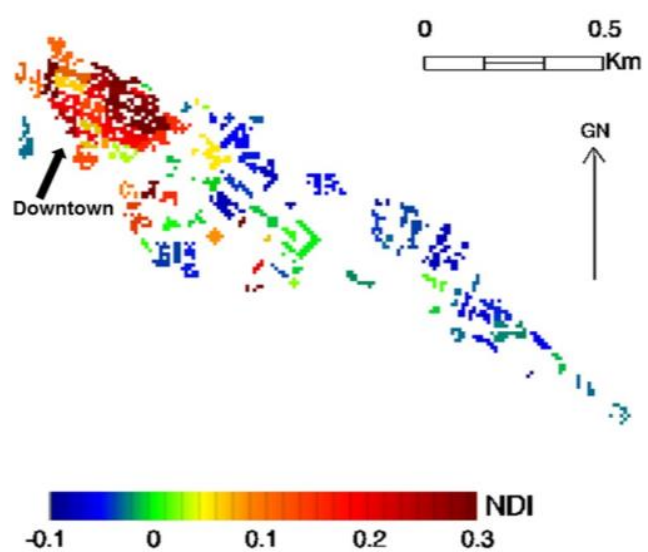

Figure 1: NDI map over Amatrice town obtained from pre- and post-seismic Sentinel-2 image

Actual NDI values range from -0.080 and 0.311 , with the highest values centred on the historical centre of Amatrice town, where most severe damage were registered (Azzaro et al. 2016).

The KLD parameter is defined as:

$$
\begin{aligned}
K L D_{i} & =\frac{\left(P R E_{i}-P O S T_{i}\right)^{2}+\operatorname{Var}\left(P R E_{i}\right)+\operatorname{Var}\left(P O S T_{i}\right)}{2} \times \\
\left(\frac{1}{\operatorname{Var}\left(P R E_{i}\right)}+\frac{1}{\operatorname{Var}\left(P O S T_{i}\right)}\right)-2 & \text { (2) }
\end{aligned}
$$

Where PRE $i$ and POSTi are the same parameters in the Eq. 1, and $\operatorname{Var}(P R E i)$ and $\operatorname{Var}(P O S T i)$ are their variances within the i-th polygon. Nominally, the KLD parameter has the same behaviour of NDI: KLD increasing values correspond to increasing damage level. The KLD values, calculated from the two Sentinel-2 optical images, range from 0.0 and 8.5.

The MI index measures the correlation between pre- and post-seismic images (see Equation 3).

$$
M I_{i}=-\ln \left(1-r_{i}^{2}\right) * 0.5
$$

Where $r_{i}^{2}$ is the correlation between the pre- and post-seismic pixels within each polygon. MI is related to the spatial arrangement of the pixels, and it is inversely proportional to the damage grade. For the present case study, its values range from 0.0 to 3.0.

As far as the SAR data is concerned, the KLD feature is derived in the same way as the optical data, while the ICD can be computed on the base of the Pearson Correlation coefficient ( $\rho i)$ estimated on the pre-seismic SAR image pair ICpre and on the co-seismic SAR image pair ICcos. These two intermediate output are then used to obtain the ICD:

$$
I C D_{i}=I C p r e-I C \cos
$$

Each feature provides a qualitative assessment of the damage distribution within the town. In order to achieve a damage map useful in the rescue operations, the unsupervised algorithm FST (Romaniello et al. (2016)) and based on these satellite features, has been employed; the scheme of the algorithm is shown in the Fig. 2.

The algorithm is able to use all change features, both from optical and SAR data, analyzing the distribution of values of each parameter. The entire ranges of values of each parameter, obtained excluding the outliers, are divided into three intervals corresponding to three damage levels. This classification is repeated $n$-times (in the present analysis, $\mathrm{n}$ is 
equal to 21) varying the width of the three intervals at each iteration.

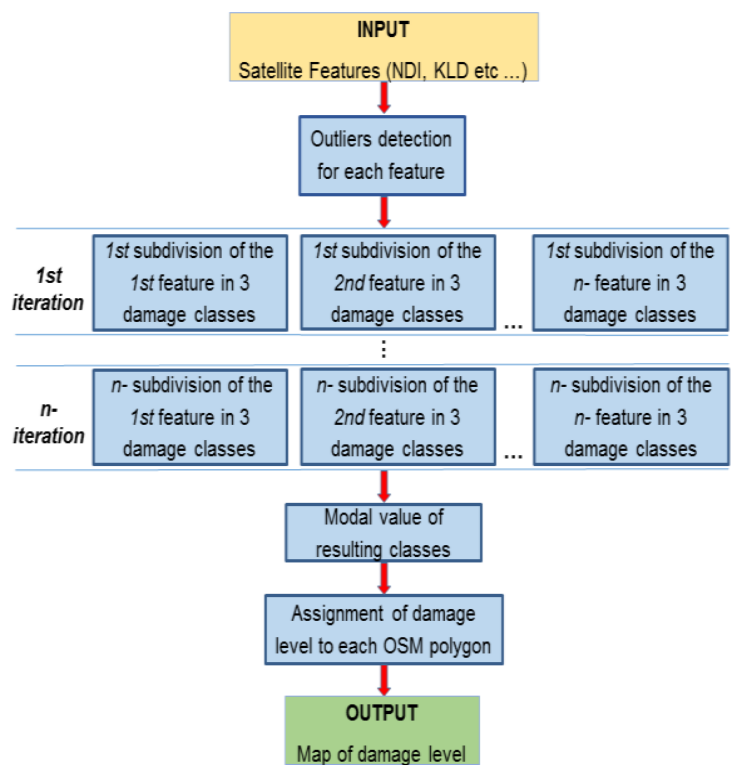

Figure 2: FST method block scheme

So, we obtain several damage level estimations for each polygon (specifically, 21 iterations $\times 5$ features). After this operation, in order to assign the final damage level (low, medium and high) to a polygon, the modal value of all previous estimations is considered for that polygon. The output is a map of the damage level occurred in Amatrice.

It is worth noticing that the damage classification (low, medium and high) is based on a relative scale. Romaniello et al. (2016) have shown the correlation between this kind of damage classification and the collapse ratio, defined as the ratio between the number of collapsed buildings and the total number of buildings within a polygon. Therefore, high damage can indicate many collapses within a city block.

\section{RESULTS}

A damage-retrieved map can be obtained by using both optical and SAR satellite images as input for the FST method. The data fusion approach showed the best damage assessment. The resulting damage map is reported in the Fig. 3. From the classification, 86 polygons are associated to the lowest damage grade, 12 to the medium and 14 to the highest.

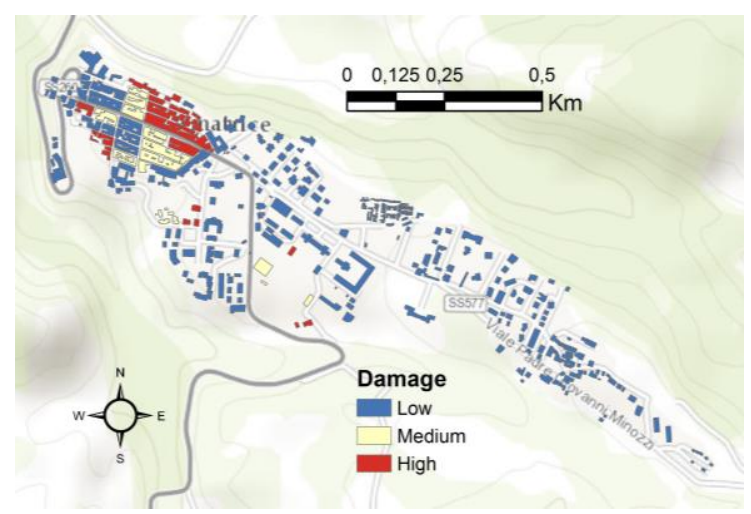

Figure 3: Damage classification by using all change features (both optical and SAR) over Amatrice town

The most affected area is the historical centre of the town and in particular the West side as visible in Fig. 4.

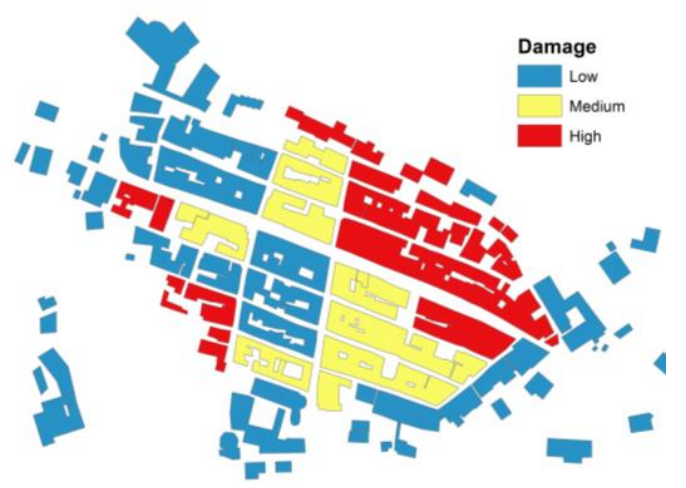

Figure 4: Damage classification in the historical centre. 
The data fusion approach has overcome false alarms problems occurred in the single sensor approach for several buildings. In particular, the analysis performed using only optical features has led to false positive cases recognized by the comparison with a very high resolution VHR image acquired after the seismic event (Fig. 5).

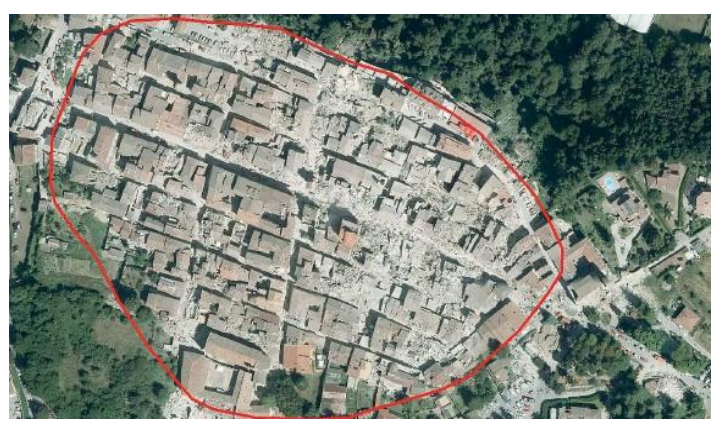

Figure 5: Amatrice post- earthquake damage.

In the classification maps reported in Fig. 3 and Fig. 4 the most affected area is the West side of the historical center with several collapsed buildings. This confirms the soundness of the FST method. Nevertheless, a quantitative assessment will be performed in the next future to draw much more detailed conclusion on the accuracy of the derived products.

\section{DisCUSSION}

The proposed method is a quite robust approach being based on unsupervised classification and employing several change features, both from optical and SAR data.

Furthermore, this method, being based on city blocks areas, is reliable with respect to changes not related to earthquake, because we can guess that modifications caused by temporal changes are small (within the blocks) if compared with the changes due to such catastrophic event. In other words, these sources of errors are averaged within the blocks.
Moreover, it is worth noticing that the proposed damage classification is on a relative scale. Indeed, the method identifies always three classes of damage associated with the low, medium and high grades of changes for the specific regions we are analyzing. On the one hand, this characteristic can be considered as a limitation of the algorithm, but from an operational point of view, we think that during rescue activities it is important to know where to intervene promptly, independently from the absolute value of damage.

Finally, a comparison with the macroseismic survey will be done, as soon as a complete survey will be available.

\section{ACKNOWLEDGEMENTS}

The research leading to these results has received funding from the European Union's Seventh Framework Programme (FP7/20072013) under grant agreement number 606738, APhoRISM project.

\section{REFERENCES}

Azzaro, R., and the QUEST Working Group (2016). "The 24 August 2016 Amatrice earthquake: macroseismic survey in the damage area and EMS intensity assessment", Ann. Geophys., vol. 59, no. 5 .

Bignami, C., Chini, M., Pierdicca, N. and Stramondo, S. (2004). Comparing and combining the capability of detecting earthquake damage in urban areas using SAR and optical data. Proceedings of IEEE IGARSS, 1, pp. 55-58.

Brunner, D., Lemoine, G. and Bruzzone, L. (2010). "Earthquake damage assessment of buildings using VHR optical and SAR image- 
ry", IEEE Trans. Geosci. Remote Sens., vol. 48, no. 5, pp. 2403-2420.

Chini, M., Piscini, A., Cinti, F.R., Amici, S., Nappi, R., De Martini, P.M. (2013). The 2011 Tohoku (Japan) Tsunami inundation and liquefaction investigated through optical, thermal, and SAR data. IEEE Trans. Geosci. Remote Sens., 10, 347-351.

Hoffman, J., Roth, A. and Voigt, S. (2004). Interferometric displacement and damage assessment for the 2003 Bam earthquake. In Proceedings of the 2004 Envisat and ERS Symposium, Salzburg, Austria, 6-10 September 2004, ESA SP-572.

Ingram K., Knapp E., and Robinson J. W. (1981). Change detection technique development for improved urbanized area delineation, technical memorandum CSCITM-81/6087, Computer Sciences Corporation, Silver Springs, Maryland, U.S.A.

Jenson J. R. (editor) (1983). Urban/suburban land use analysis. Manual of Remote Sensing, Vol. 2, second edition (Falls Church, Virginia: American Society of Photogrammetry), pp: 1571-1666.

Kullback, S.; Leibler, R. A. (1951). On Information and Sufficiency. Ann. Math. Statist. 22, no. 1, 79--86. doi:10.1214/aoms/1177729694. http:// projecteuclid.org/euclid.aoms/1177729 694.

Matsuoka, M. and Yamazaki, F. (2004). Use of satellite SAR intensity imagery for detecting building areas damaged due to earthquakes. Earthquake Spectra, 20, pp. 975-994.

Romaniello, V., Piscini, A., Bignami, C., Anniballe, R. and Stramondo, S. (2016). Earthquake damage mapping by using remotely sensed data: the Haiti case study, Journal of Applied Remote Sensing, (submitted).

Stramondo, S. Bignami, C., Chini, M., Pierdicca, N., Tertulliani, A. (2006). Satellite radar and optical remote sensing for earthquake damage detection: results from different case studies. International Journal of Remote Sensing, 27, 4433 4447.

H. Xie, L. E. Pierce, and F. T. Ulaby (2003). "Mutual information based registration of SAR images," in Proc. IEEE IGARSS, Toulouse, France, Jul. 2003, pp. 4028-4031.

Yonezawa, C. and Takeuchi, S. (2001). Decorrelation of SAR data by urban damage caused by the 1995 Hoyogoken-Nanbu earthquake. International Journal of Remote Sensing, 22, pp. 15851600. 